\title{
Lumen
}

Selected Proceedings from the Canadian Society for Eighteenth-Century Studies

\section{Absences et présences de l'art du voyage dans la France du XVIII $^{\mathrm{e}}$ siècle}

\section{Gabor Gelléri}

Volume 34, 2015

URI : https://id.erudit.org/iderudit/1028511ar

DOI : https://doi.org/10.7202/1028511ar

Aller au sommaire du numéro

Éditeur(s)

Canadian Society for Eighteenth-Century Studies / Société canadienne d'étude du dix-huitième siècle

ISSN

1209-3696 (imprimé)

1927-8284 (numérique)

Découvrir la revue

Citer cet article

Gelléri, G. (2015). Absences et présences de l'art du voyage dans la France du XVIII ${ }^{\mathrm{e}}$ siècle. Lumen, 34, 55-69. https://doi.org/10.7202/1028511ar 


\title{
Absences et présences de l'art du voyage dans la France du XVIII ${ }^{\mathrm{e}}$ siècle
}

\author{
Gabor Gelléri \\ Aberystwyth University
}

C'est probablement Paul Hazard avec son ouvrage La crise de la conscience européenne $e^{1}$ qui a ancré la conviction que les voyages représentent un élément essentiel dans la pensée française à partir du début de la «crise » qu'il décrit. L'article fondateur de René Pomeau «Voyage et Lumières » souligne lui aussi ce fait, mais introduit un autre élément important: l'ambivalence profonde de l'attitude française face aux voyages à cette époque ${ }^{2}$. Malgré la fascination pour les voyages et les voyageurs, et malgré le fait que la grande majorité des auteurs de l'époque se nourrit de la littérature de voyages ${ }^{3}$, il existe une méfiance durable face à cette forme d'écriture.

Mon intention ici n'est pas de rouvrir le débat sur cette méfiance. L'angle que je voudrais présenter n’a pas encore été suffisamment étudié dans ce débat: c'est celui de «l'art du voyage », l'ars apodemica.

1. Paul Hazard, La crise de la conscience européenne, 1680-1715. Paris, Fayard, 1961 (éd. originale Paris, Boivin, 1935); surtout le premier chapitre: «De la stabilité au mouvement», p. 3-25.

2. René Pomeau, "Voyages et Lumières dans la littérature française du dixhuitième siècle », Studies on Voltaire and the Eighteenth Century, LVI, 1967, p. 12691289.

3. Il est certainement remarquable que parmi les bibliothèques des «grands noms " de l'époque, la plus faible en titres de voyage (avec à peine $1 \%$ du total) soit celle d'un des rares auteurs qui aient vraiment voyagé: Montesquieu (voir le Catalogue de la bibliothèque de Montesquieu, publié par Louis Desgraves. Genève / Lille, Droz / Giard, 1954). Sur l'importante présence des livres de voyage dans les bibliothèques d'autres figures majeures de l'époque, voir Michèle Duchet, Anthropologie et histoire au siècle des Lumières: Buffon, Voltaire, Rousseau, Helvétius, Diderot, Paris, Maspero, $1995[1971]$. 
L'apparition de cet ensemble de textes analysant les pratiques de déplacement en vigueur, et esquissant l'horizon d'attente d'une pratique idéale, est un phénomène crucial de l'époque moderne. Ce corpus a été analysé du point de vue de son rôle de précurseur des sciences humaines et sociales, comme une série de discours se rapportant à une pratique sociale et intellectuelle, mais également en tant que tradition textuelle et intellectuelle sui generis, avec des communications intertextuelles et souvent interpersonnelles remarquables ${ }^{4}$.

Il n'existe aucun aperçu général de la production française de l'époque, et l'objectif de cet article ne peut pas être de combler cette lacune. Il est possible de trouver des réflexions sur le sujet dans certaines monographies consacrées à des phénomènes de voyage particuliers, notamment dans celle de Gilles Bertrand sur le voyage d'Italie, et dans cette somme qu'est le livre de Daniel Roche, Humeurs vagabondes $^{5}$. Je propose plutôt d'observer, à travers un parcours de la production française dans ce genre, depuis le début du siècle jusqu'aux années de la Révolution, la façon dont l'attitude ambiguë face aux voyages se traduit dans la production apodémique française.

Commençons par remonter dans le temps, et par formuler ici une rapide observation concernant l'idée de «crise» et son lien avec les voyages. Selon Paul Hazard, l'absolutisme en «bonne santé » se caractériserait par un refus du voyage, et les opinions de cette nature sont pléthore à cette époque. Cependant - et dans les nombreuses critiques adressées à cet ouvrage, ce point a été moins traité -, il semblerait que le refus du voyage que Paul Hazard associe à l'idée d'absolutisme soit un peu exagéré. La soif de connaissances et d'aventures est tout à fait vivante dans la France des années 1660-1670. Le régime louisquatorzien a «ses » propres voyages et voyageurs. La Boullaye le Gouz

4. Normand Doiron (L'art de voyager. Le déplacement à l'époque classique, Paris / Sainte-Foy, Klincksieck / Presses de l'Université de Laval, 1995) se concentre avant tout sur l'aspect rhétorique de cette production; son caractère de précurseur des méthodologies scientifiques et l'importance des rapports interpersonnels sont soulignés avant tout par Justin Stagl (après plusieurs articles, voir en particulier A History of Curiosity, Travel theory 1550-180o, Chur, Harwood Academic, 1995).

5. Gilles Bertrand, Le Grand Tour revisité: pour une archéologie du tourisme. Le voyage des Français en Italie, milieu XVIII ${ }^{e}$ siècle-début XIX $X^{e}$ siècle, Rome, École Française de Rome; Daniel Roche, Humeurs vagabondes. De la circulation des hommes et de l'utilité des voyages, Paris, Fayard, 2003. 
ou La Loubère ${ }^{6}$ sont des voyageurs «soutenus » par le régime; un peu plus tard, le père Tachard voyage, puis publie son récit par ordre du $\mathrm{roi}^{7}$; tandis que Bernier reçoit toute une liste de suggestions/instructions de Chapelain qui souhaite diriger, " policer» ses observations ${ }^{8}$.

Il ne nous semble donc pas possible d'affirmer que l'absolutisme soit hostile à toute idée de voyage; mais - comme l'exemple de Bernier ou celui des voyages par ordre du roi le montrent - le régime fait de son mieux pour contrôler le flux d'informations concernant d'autres cultures et modes de vie. Et même dans ce texte fameux si souvent cité comme preuve de la méfiance envers les voyages, La tortue et les deux canards de La Fontaine, n'est-ce pas plutôt l'ambition de l'homme moyen d'aller voir «mainte république, maint royaume, maint peuple» qui est ridiculisée, plutôt que l'idée même de voyager ${ }^{9}$ ?

Formulons d'abord quelques remarques générales sur la production apodémique française du XVIII ${ }^{\mathrm{e}}$ siècle. La bibliographie publiée par Justin Stagl et ses collègues permet une première évaluation quantitative $^{10}$ : dans celle-ci, ce sont les textes allemands qui dominent avant tout (plus de $50 \%$ des textes du XVIII ${ }^{\mathrm{e}}$ siècle), et nous voyons légèrement plus de textes anglais que français. Cependant deux observations s'imposent. Tout d'abord, de nombreux textes anglais du XVIII' siècle y manquent, tandis que certains des textes français que nous y trouvons sont problématiques. Ainsi, par exemple, la dissertation de Gros de Besplas ne porte pas sur le voyage comme pratique, mais uniquement

6. François La Boullaye le Gouz, Les voyages et observations du sieur de -, gentilhomme angevin..., Paris, Gervais Clousier, 1653; Simon de La Loubère, Du Royaume de Siam, Paris, Coignard, 1691.

7. Guy Tachard, Voyage du Siam des Pères jésuites, envoyés par le Roy aux Indes et à la Chine, Paris, A. Seneuze, 1686: «C'est par votre commandement exprès que je rends compte au public d'un voyage que j'entrepris par votre ordre pour l'utilité de toutes les nations» («Epistre au Roy», Aii- $\mathrm{v}^{0}$ ).

8. Pour les lettres de Chapelain, voir Louis de Lens, «Les correspondants de François Bernier pendant son voyage dans l'Inde. Lettres inédites de Chapelain », Mémoires de la Société nationale d'agriculture, sciences et arts d'Angers, Angers, Éditions de l'Ouest, 1872 , t. XV, p. 129-176.

9. Livre X, fable 2. L'analogie «Ulysse en fit autant» suivie de la remarque «On ne s'attendait guère / de voir Ulysse en cette affaire » montre clairement que le voyage est l'apanage d'une élite seulement, de même que le droit de discuter et d'imiter les gestes des «Ulysse».

10. Justin Stagl, Klaus Orda et Christian Kämpfer, Apodemiken. Eine räsonnierte Bibliographie der reisetheoretischen Literatur des 16., 17. und 18. Jahrhunderts, Paderborn, Schöningh, 1983. 
sur les bénéfices apportés par la lecture des voyages ${ }^{11}$. Ces deux défauts masquent l'infériorité numérique de la production française non seulement vis-à-vis de la production allemande, mais aussi vis-à-vis de la production anglaise.

Le retard français pourrait s'expliquer, entre autres, par la nature des textes et par leur lien avec certaines pratiques sociales. En Allemagne, c'est avant tout la culture universitaire qui explique la fréquence de ce type de texte: l'utilité (et parfois l'inutilité) des voyages figure parmi les sujets les plus souvent traités dans les exercices rhétoriques. En Angleterre, où, malgré une abondante littérature qui le condamne ou le parodie, le voyage fait partie intégrante de l'éducation des jeunes aristocrates et des familles bourgeoises aisées, presque tout ouvrage pédagogique des $\mathrm{XVII}^{\mathrm{e}}$ et $\mathrm{XVIII}^{\mathrm{e}}$ siècles se voit dans l'obligation de consacrer quelques pages aux best practices du voyage pédagogique. Cette tradition du voyage pédagogique est, bien entendu, également très vivante en Allemagne.

La culture des dissertations universitaires est, pour l'essentiel, absente en France. Quant aux ouvrages pédagogiques, l'apparition du voyage parmi les thèmes traités est loin d'être aussi systématique qu'en Angleterre, et même lorsque c'est le cas, les passages que ces ouvrages consacrent aux voyages sont peu connus, à l'exception du chapitre dans l'Émile de Rousseau. Il est sans doute symptomatique que le Spectacle de la nature de l'abbé Pluche, qui est pourtant le best-seller pédagogique absolu de l'époque, contienne une dissertation sur les voyages ${ }^{12}$, mais celle-ci est peu citée à l'époque, et ignorée par la quasi-intégralité de la critique scientifique. Le texte français le plus complet de cette nature, une dissertation imprimée en Italie, présente de nombreux éléments obscurs, notamment l'identité précise de son auteur et de ses destinataires; et ce texte, qui survit en un seul exemplaire en France, ne semble pas non plus avoir eu d'impact ${ }^{13}$.

11. Joseph Marie André Gros de Besplas, De l'utilité des voyages relativement aux sciences et aux mours, discours prononcé par M. l'abbé Gros de Besplas... à sa réception, en qualité d'associé, à l'Académie de Bésiers, au mois d'août 1762, avec des réflexions sur les voyages, Paris, Berthier, 1763.

12. Noël-Antoine Pluche, Le spectacle de la nature, ou Entretiens sur les particularités de l'histoire naturelle [...]. Tome septième: Contenant ce qui regarde l'homme en société, Paris, Veuve Estienne et fils, 1747, p. 448-486: «L'esprit des voyages et du commerce».

13. Discours sur l'utilité des voyages, s.l., s.n., 1780 (seul exemplaire connu en France: Vincennes, Bibliothèque du Service historique de la Défense, 4J9). 
Au-delà de l'infériorité quantitative de la production française et de son caractère souvent marginal et méconnu, il existe encore d'autres domaines où une relative «absence » de l'art du voyage mérite d'être soulignée.

Le premier texte que nous souhaitons prendre en compte est la définition du mot «Voyage » dans l'Encyclopédie, souvent citée comme l'idéal du «voyage encyclopédique». Dans cette définition, déjà peu originale, se retrouve la méfiance face à la littérature de voyages déjà évoquée plus haut et soulignée par René Pomeau. Y manque en revanche une manifestation du programme intellectuel de l'Encyclopédie, au-delà de la collecte d'informations.

Cet article «Voyage» est signé par le chevalier Jaucourt, le factotum prolixe mais peu original de l'équipe éditoriale. Et, comme dans le cas de tant d'autres définitions qu'il a rédigées avec ses collaborateurs, il se contente de reprendre les idées reçues de l'époque: on y retrouve les mêmes éléments, dont la collecte d'informations, qui figurent dans tous les dictionnaires depuis le dernier tiers du $\mathrm{XVII}^{\mathrm{e}}$ siècle. L'Encyclopédie innove uniquement par l'ajout des manufactures à la liste des domaines d'observation. Et même la citation de Strabon qui illustre la méfiance vis-à-vis des voyages se retrouve dans la plupart des dictionnaires français, depuis la deuxième édition du dictionnaire de Furetière, établie par Basnage de Bauval ${ }^{14}$.

Il faudrait peut-être plutôt se demander pourquoi l'Encyclopédie ne contient pas d'article plus détaillé et original consacré à ce phénomène si important pour l'époque, et pourquoi la rédaction de cet article a été confiée à un auteur connu pour son peu d'originalité (On aurait certainement préféré une définition de la plume de Montesquieu qui, lui, a voyagé !). Cependant, il faut noter qu'au moment de la rédaction de l'article, aucun des auteurs principaux de l'Encyclopédie n'a fait de grands voyages (les voyages de Diderot sont ultérieurs).

À ce propos, il faut évoquer, justement, la préface du Voyage de Hollande de Diderot ${ }^{15}$ : un texte certainement plus développé, plus

14. «Strabon dit que tout homme qui conte ses voyages est menteur» (Dictionnaire universel [...] par feu messire Antoine Furetière [...] $2^{e}$ édition revue, corrigée et augmentée par M. Basnage de Bauval, Rotterdam, R. Leers, 1702, vol. II, p. 1102.

15. Denis Diderot, Voyage en Hollande, avec introduction et notes d'Yves Benot, Paris, Maspero, 1982: Préliminaire: Des moyens de voyager utilement. Le texte date de 1774 . 
ambitieux, rédigé par une autorité intellectuelle incontestable. Mais qui n'est pas, non plus, sans contradictions. Tout d'abord, ce texte est, dans le domaine de l'art du voyage, d'un archaïsme remarquable. Devant l'incertitude des observations personnelles dans les domaines où une connaissance approfondie serait nécessaire, Diderot suggère un programme dont l'élément majeur est la fréquentation des experts sur place, et la consultation d'ouvrages spécialisés. Or, il ne fait là que répéter un principe de base de l'art du voyage depuis la naissance du genre dans le dernier tiers du Xvi ${ }^{e}$ siècle. L'incontournable Juste Lipse n'a-t-il pas suggéré que le voyageur passe son temps dans un triangle d'occupations, entre livres à lire, personnes à voir, et choses à visiter, avec une nette priorité accordée aux deux premières ${ }^{16}$ ?

Le voyage même de Diderot porte le titre d'«Application des précédents à la Hollande». Mais, comme le souligne Yves Benot, d'une façon remarquable, cette étape suivante, à savoir appliquer la méthode décrite dans la Préface à son propre voyage, n’a pas lieu ${ }^{17}$. Par conséquent, cette préface reste un texte isolé, relativement peu connu - et semble, comme telle, appartenir également à la liste des «absences » de l'art du voyage.

Le concours académique est une production typiquement française qui représente, dans une certaine mesure, un pendant français à la production typiquement allemande de la dissertation universitaire. Le concours de l'Académie de Lyon en 1787 sur le sujet «Les voyages peuvent-ils être considérés comme un moyen de perfectionner l'éducation?» en est l'exemple le plus important - et le plus surprenant ${ }^{18}$.

Le jury du concours ouvre son commentaire sur un jugement d'ensemble de la production, et déclare d'emblée que la qualité de la très grande majorité des discours est lamentable, reflet - d'après le jury - du 1619.

16. Voir Le choix des epistres de Juste Lipse, traduction d'Antoine Brun, Lyon, s.n.,

17. Denis Diderot, Voyage en Hollande, op. cit., p. 12.

18. Extrait des discours qui ont concouru pour le prix que l'Académie des sciences, des belles-lettres et des arts de la ville de Lyon a adjugé à M. Turlin, avocat du Parlement de Paris, sur cette question: «Les voyages peuvent-ils être considérés comme un moyen de perfectionner l'éducation? ", Lyon, s.n., 1787. Ce concours a été étudié également, sous un angle différent, par Daniel Roche, Humeurs vagabondes, op. cit., p. 77-87. 
nombre de clichés qui circulent sur la question ${ }^{19}$. Ce n'est pas la question et le sujet mêmes qu'ils condamnent - suggérée par un père de famille, la question a bien été appréciée par l'Académie -, mais c'est la réflexion française sur le sujet qui s'est avérée décevante.

Le jury a ainsi eu bien de la peine à identifier deux textes auxquels décerner le prix et l'accessit. L'attribution de l'accessit a été justifiée par le fait que l'auteur, à côté de nombreux lieux communs, évoque un sujet nouveau: l'importance du voyage dans l'éducation physique. Sans que l'idée soit entièrement nouvelle dans la littérature sur les voyages, elle est en train d'émerger à cette époque ${ }^{20}$.

Le jury s'est vu ensuite obligé de donner le prix au seul texte, selon eux, vraiment de qualité: le discours de Turlin, avocat du parlement de Paris, qui signerait là son unique contribution littéraire. L'hésitation du jury est due au fait que Turlin, loin de parler en faveur des voyages, les condamne ${ }^{21}$. L'essentiel de son programme se trouve dans la phrase d'ouverture spectaculaire: «Les voyages sont bons en eux-mêmes et il faut l'interdire $[s i c]$ à notre jeunesse; c'est ainsi que, dans un siècle pervers, les choses les plus saines se corrompent ${ }^{22}$. L La crise du voyage est, pour Turlin, le signe évident d'une crise plus générale.

Ce texte mérite certainement notre attention en raison de son appartenance à la tradition particulière des textes contre les voyages tradition dans laquelle nous trouvons par exemple le Quo vadis de l'évêque Joseph Hall, et la Lettre sur les voiages du suisse Béat de Muralt ${ }^{23}$. Cette tradition, jamais étudiée dans son ensemble, est du plus grand intérêt; cependant nous nous concentrons cette fois sur l'apport "positif», sur les véritables programmes de voyage. Le fait que la très grande majorité des participants du concours régurgitent des

19. Dans Extrait des discours, op. cit., les pages 10-13 résument les rares idées d'une vingtaine de discours, avec la remarque du jury: "Voilà ce qu’on a pu extraire d'environ vingt discours qui rendent ce concours plus riche en apparence qu'en réalité» (p. 13).

20. Georges Vigarello, Le corps redressé: histoire d'un pouvoir pédagogique, Paris, J.-P. Delarge, 1978.

21. Soulignons ici le parallèle avec cet autre vainqueur «problématique» d'un concours académique, le Discours sur les sciences et les arts de Rousseau.

22. Extrait des discours, op. cit., p. 47.

23. Joseph Hall, Quo vadis? Or a Just Censure of Travell, as It Is Commonly Undertaken by the Gentlemen of our Nation, Londres, Edward Griffin for Henry Featherstone, 1617; Béat Louis de Muralt, Lettres sur les Anglois et les François et sur les voiages, Genève, Fabri et Barrillot, 1725 . 
topoi creux, et que le seul travail de qualité profite de cette occasion pour évoquer une crise de la civilisation moderne, indique que le domaine des compétitions académiques relève également davantage de l'absence que de la présence de l'art du voyage en France.

Faut-il parler d'un vide français des arts de voyage? Face à cette impressionnante liste d'absences, et sans viser l'exhaustivité, je souhaite souligner l'existence d'un certain nombre de véritables programmes apodémiques français.

Le premier est ce texte si peu apprécié par la critique moderne qu'est le programme de voyage dans Émile. On le juge traditionaliste et conventionnel, et on néglige la tradition textuelle à laquelle il appartient ${ }^{24}$. On souligne également - et certainement à juste titre - le côté extrêmement sexué de ce programme. Yaël Schlick a parfaitement présenté les contradictions entre les deux moitiés du livre V d'Émile, la partie sur Sophie et celle sur les voyages ${ }^{25}$. Le programme d'éducation que Rousseau suggère pour Sophie n’a certainement pas changé la tradition dans la mesure où le voyage d'éducation n'est jamais suggéré pour les jeunes filles.

Et, certes, une bonne partie du programme de voyage que Rousseau formule est conventionnelle. Mais ceci n'est pas une raison suffisante, nous semble-t-il, pour l'écarter pour autant: être conventionnel est, en fait, être dans l'esprit de l'ars apodemica. Le genre des dissertations sur le voyage est extrêmement rigide, et il a à peine changé depuis ses origines dans la Renaissance tardive ${ }^{26}$.

Le programme dans Émile apporte cependant des nouveautés - et celles-ci sont d'une envergure considérable. La première est la suggestion, si typique de Rousseau, de voyager à pied - suggestion qui apparaît aussi à travers les promenades de Saint-Preux, dans les nombreux moments de promenade des Confessions et, plus tard, dans les Rêveries

24. Ainsi Sarga Moussa, dans l'article «Voyage» du Dictionnaire de Jean-Jacques Rousseau (Raymond Trousson et Frédéric S. Eigeldinger [dir.], Paris, Honoré Champion, 1996, p. 934-936), n'examine qu'en dernier lieu ce programme de voyage, et sans la moindre allusion à l'appartenance de ce texte à la tradition apodémique.

25. Yaël Schlick, Feminism and the Politics of Travel after the Enlightenment, Lewisburg, Bucknell University Press, 2012; voir surtout le premier chapitre, «The Sex of Travel: Sexual Contract and Enlightenment Travel in Jean-Jacques Rousseau and Mary Wollstonecraft», p. 19-50.

26. Souligné par Justin Stagl, A History of Curiosity, op. cit.; voir ici surtout l'introduction, p. 1-46. 
du promeneur solitaire. Cet élément sera reflété par la suite dans la pratique de plusieurs générations de voyageurs et dans la parution de toute une avalanche d'ouvrages, Promenades et autres Fussreisen.

L'autre innovation majeure de Rousseau se situe dans la question de la finalité du voyage. Débattre des bénéfices que le voyage apporte est certes traditionnel, particulièrement dans les livres d'éducation: pour l'éducateur, le résultat du voyage est aussi (voire plus) important que la question de la «méthode» à suivre lors du voyage. Mais le bénéfice que Rousseau espère tirer des voyages est inédit, et radical.

Cette conception du voyage est en fait intimement liée à l'idée de «se fixer» que Jean Starobinski, entre autres, a souligné comme motif central de l'œuvre de Rousseau ${ }^{27}$. Le voyage d'Émile s'achève sur un dialogue hypothétique: le précepteur demande à Émile ce qu'il a appris de ses voyages. Si la méthode est correcte, dit Rousseau, Émile répondra : «À quoi je me fixe? à rester tel que vous m'avez fait être ${ }^{28}$. »

Le voyage aura bien permis de connaître les pays parcourus, et surtout les hommes. Mais l'objectif essentiel de tout le voyage est de retourner, retourner à soi-même, à ce que Georges Van Den Abbeele appelle «the self as domus ${ }^{29} »$. Certes, tout programme de voyage, à toutes les époques, insiste sur la nécessité de ne pas abandonner ses valeurs au cours du voyage, de ne pas y perdre son identité nationale ou religieuse. Mais Rousseau va plus loin, à notre sens, que de simplement - comme le suggère Sarga Moussa - «faire le détour par autrui pour apprendre à se connaître ${ }^{30} »$.

Rousseau espère que le voyage d'Émile donnera lieu à un changement bien précis. Ce changement est la naissance du désir de ne pas changer, ne plus changer, ce qui revient à «se fixer » - ce mot est même présent dans la réponse d'Émile. La quasi-contradiction logique (le changement survenu est de ne plus vouloir changer) est typique de Rousseau; et c'est de cette nature frôlant la contradiction que la

27. Jean Starobinski, Jean-Jacques Rousseau: La transparence et l'obstacle, Paris, Plon, 1957, p. 63-72.

28. Jean-Jacques Rousseau, Émile ou De l'éducation, La Haye, Jean Neaulme, 1762. Nous citons d'après les (Euvres complètes, Paris, Seuil, 1971, vol. 3, p. 306-321.

29. Georges Van Den Abbeele, Travel as Metaphor, from Montaigne to Rousseau, Minneapolis / Oxford, University of Minnesota Press, 1992, p. 17.

30. Dictionnaire de Jean-Jacques Rousseau, op. cit., p. 936. 
conception du voyage de Rousseau tire toute sa force; et l'impact de cet aspect-ci sera, lui aussi, important.

Le deuxième domaine de "présence » que je voudrais évoquer est la reconnaissance croissante en France de l'importance de l'activité marchande, non seulement du point de vue économique, mais aussi intellectuel. Nous la retrouvons également dans le contexte spécifique de l'art du voyage. La bibliographie Apodemiken contient l'Histoire du commerce et de la navigation des anciens de l'évêque Pierre-Daniel Huet, tout en ajoutant qu'elle ne contient « rien de proprement apodémique $^{31} »$. En revanche, d'autres titres évoquant le voyage marchand n'ont pas que des similarités superficielles avec l'art du voyage. Fait quelque peu surprenant, c'est l'abbé Pluche, bien connu comme vulgarisateur d'histoire naturelle mais moins comme commentateur social, qui évoque le mieux cette «interconnexion» de savoirs entre les groupes sociaux. On peut légitimement suggérer que cette appréciation du tiers état s'explique par les origines de Pluche, issu des milieux très modestes de Reims.

Un chapitre du Spectacle de la nature porte le titre «Sur l'esprit des voyages et du commerce $\aleph^{32}$. Au cours des dialogues sur l'utilité des voyages, un père de famille noble demande conseil à un père de famille commerçant sur la méthode à suivre pour le voyage de son fils; et l'on insiste à plusieurs reprises sur le savoir-faire marchand. Que l'apport spécifique des voyageurs marchands soit aussi nettement reconnu, et même recommandé aux familles nobles, est certainement une nouveauté.

En même temps, sur de nombreux autres points, Pluche est traditionaliste, à plus d'un titre. Il répète, lui aussi, les clichés du genre sur le comportement à suivre et les observations à réaliser. Il insiste particulièrement sur l'aspect religieux du voyage. Il ne s'agit pas de transformer le voyage en pèlerinage; mais les conseils traditionnels sur la santé des voyageurs (les regimina) sont accompagnés ici de conseils pour la protection de l'esprit, de la foi. Nous trouvons des conseils similaires dans la littérature apodémique anglaise, où il s'agit de se protéger des dangers du papisme. Pour Pluche, le danger est bien sûr

31. Justin Stagl, Klaus Orda et Christian Kämpfer, Apodemiken, op. cit., p. 55 : «Nichts eigentlich Apodemisches enthält.»

32. Pluche, Le spectacle de la nature, op. cit., t. 7, p. 448-486. 
opposé, et le remède à toute épreuve pour maintenir la foi catholique est d'avoir toujours avec soi un exemplaire de l'Imitation de JésusChrist. Mais les dangers ne viennent pas uniquement des autres religions : il met en garde son lecteur contre « une philosophie orgueilleuse, qui, malgré la fragilité de la raison dans les choses naturelles, prétend établir la raison juge de ce qu'il faut croire et espérer ${ }^{33}{ }$. Cet art du voyage a de forts accents anti-philosophiques.

La même interconnexion avec le domaine de l'art de voyage apparaît également dans la production des marchands eux-mêmes. Il existe toute une tradition de manuels de marchands, avec des conseils divers sur une variété de domaines dont la plupart ne relève pas de «l'art du voyage », mais de l'activité commerçante. Cependant un Almanach du commerce et des voyageurs, publié chez Duchesne probablement en 1774 , a pour sous-titre «avec des instructions pour voyager utilement, commodément et agréablement ${ }^{34} »$. Il faut reconnaître que cette section n'est guère novatrice, et nous la citons avant tout pour souligner la connexion marchande: elle contient quelques lieux communs apodémiques et des conseils pratiques. Parmi ces derniers, notons un élément qui paraît être unique: est donné en Annexe un tableau pour estimer ce qu'il est judicieux de dépenser lors d'un voyage, en fonction des revenus réguliers du voyageur.

Un Discours sur l'utilité des voyages, publié en 1780, probablement à Bologne, et entièrement inconnu de la critique, est attribué par une note manuscrite présente sur le seul exemplaire qui se trouve en France à un certain abbé Des Acharts, qui aurait été précepteur en Italie ${ }^{35}$.

Aussi méconnu que puisse être ce texte, il s'agit de l'ouvrage apodémique français le plus développé du siècle - et du seul titre, à part la compétition académique, qui soit intégralement consacré à la question des voyages. Selon ce texte, l'homme est essentiellement bon, mais il est souvent perverti par les institutions. Le voyageur doit étudier les institutions, en prenant en compte l'influence des mœurs et du climat. Il semble évident que l'auteur réalise, pour l'essentiel, un amalgame

33. Ibid., p. 457.

34. Almanach du commerce et des voyageurs contenant l'indication des villes commerçantes de l'Europe... avec des instructions pour voyager utilement, commodément et agréablement, Paris, Duchesne, 1774 [?].

35. Discours sur l'utilité des voyages, op. cit. 
pas très original, mais habile, d'idées empruntées à Montesquieu et à Rousseau, et il est probable qu'il ait également connu le texte de Pluche, sans qu'il ne cite la référence marchande.

Selon nous, l'intérêt principal du texte est ailleurs - notamment dans une comparaison ${ }^{36}$. Selon Des Acharts, l'étude des nations étrangères, si elle est effectuée de façon satisfaisante, est de la même nature que le travail du botaniste moderne: "Comme un habile Botaniste découvre dans une longue suite de plantes toutes ces variétés qui forment tout autant de classes différentes, tandis que l'œil vulgaire n'aperçoit que la multiplicité répétée d'un seul et même objet ${ }^{37}$.». Même si la botanique en tant que telle ne représente guère une nouveauté, ce n'est qu'au cours du dernier tiers du siècle qu'une pareille expression peut être imaginée: un exemple issu des sciences naturelles est utilisé pour décrire un phénomène du domaine des sciences de l'homme. Quelques années auparavant, Bernardin de Saint-Pierre regrettait encore que le vocabulaire même fasse défaut pour décrire certaines parties des plantes, tandis que le moindre détail architectural était nommé et clairement défini ${ }^{38}$. Mais ici, peut-être sous l'impact de Linné et des véhémentes réponses françaises que ses idées ont suscitées $^{39}$, ce domaine classique de la connaissance de l'homme qu'est la description d'un pays se voit associé au travail du naturaliste.

La dernière - et à notre sens la plus importante - présence, jusqu'ici tout aussi peu considérée, caractérise également le dernier tiers du siècle. Deux textes radicalement différents seront classés dans cette rubrique.

Le premier est la seule véritable réponse française à la littérature anglaise des instructions pour l'observation d'un pays. Étienne Munier, économiste et spécialiste des techniques agricoles, intendant du roi à la Généralité de Limoges, a publié une série d'instructions pour une pratique d'observation idéale. Ce type de production n'est guère nou-

36. Sur l'importance des comparaisons et des analogies dans l'écriture des voyages, voir le livre d'Alain Guyot, Analogie et récit de voyage. Voir, mesurer, interpréter le monde, Paris, Classique Garnier, 2012.

37. Discours sur l'utilité des voyages, op. cit., p. 4.

38. Henri Bernardin de Saint-Pierre, Voyage à l'Isle de France, à l'Isle de Bourbon, au Cap de Bonne-Espérance... Avec des observations nouvelles sur la nature et sur les hommes, Amsterdam / Paris, Merlin, 1773. L'essentiel de la dernière lettre est consacré à cette question.

39. Voir Pascal Duris, Linné et la France (1780-1850), Genève, Droz, 1993. 
veau - depuis l'apparition de l'ars apodemica, les descriptions «idéales » souhaitant présenter un exemple à suivre dans les méthodes d'observation et de description sont omniprésentes; on a vu Diderot se proposer le même principe en Hollande. Munier publie la liste de questions, et donne une séquence optimale de réponses possibles, à travers l'exemple de sa propre région, le pays d'Angoulême ${ }^{40}$. On retrouve là certaines pratiques connues dans les îles Britanniques (des questionnaires pour la connaissance de l'Irlande de Petty, entre autres ${ }^{41}$ ). Mais nous y voyons surtout le reflet dans le domaine apodémique d'un changement important de l'époque : l'attention de la France se focalise désormais, avant toute chose, sur la France même. Après s'être regardée pendant un siècle dans des miroirs divers mais étrangers (italien, anglais, prussien, suisse...), la France peut désormais développer une méthode idéale de gouvernement sans avoir à recourir à une quelconque médiation externe.

Ce même sujet apparaît, mais sous une forme particulière, dans le dernier texte de notre parcours: un projet de loi jacobin, déposé par un conventionnel assez peu connu, Louis Portiez. En 1794, quelques jours à peine avant la chute de Robespierre, il remet le projet intitulé «Des voyages, de leur utilité dans l'éducation » ${ }^{42}$.

D’un côté, c'est un produit typique du mouvement de «l'inculcation des valeurs républicaines ${ }^{43} »$ : le programme de voyage est entièrement conçu pour illustrer à l'intention des enfants participants (un élève de chaque classe, choisi par ses camarades) la supériorité de la France républicaine. Ils voyagent en France même pour observer l'unité du pays (plutôt idéologique que géographique, puisque ce dernier aspect n'a pas besoin d'être prouvé); et à l'étranger uniquement

40. Étienne Munier, Essai d'une méthode générale propre à étendre les connaissances des voyageurs, Paris, Moutard, 1779, 2 vol. Son nom est mal ortographié («Meunier») dans le catalogue Apodemiken.

41. Sur Petty, voir par exemple Ted McCormick, William Petty and the Ambitions of Political Arithmetic, Oxford, Oxford University Press, 2009.

42. Louis Portiez, Des voyages, de leur utilité dans l'éducation, Imprimé par ordre de la Convention nationale, s.l.n.d. [Paris, 1794]. Pour une analyse détaillée de ce document, voir notre contribution «Voyager: un programme républicain », dans Gilles Bertrand et Pierre Serna (éd.), La République en voyage 1770-1830, Rennes, Presses universitaires de Rennes, 2013, p. 221-231.

43. Voir James Leith, «The Idea of Inculcation of National Patriotism in French Education Thought, 1750-1789", dans J. D. Browning (éd.), Education in the $18^{\text {th }}$ Century, New York / Londres, Garland, 1979, p. 59-77. 
pour voir de leurs propres yeux les défauts des autres pays. L'héritage du programme de voyage formulé dans Émile est parfaitement visible: on voyage pour arriver au désir de ne pas changer. Et ne pas, ou ne plus vouloir changer est un résultat cardinal au moment de la création de ce plan, lorsque les sentiments antirépublicains menacent de prendre le dessus en France.

Trois éléments seront d'un intérêt particulier dans ce programme qui, pourtant, n'est jamais devenu réalité. Tout d'abord, il s'agit d'une proposition de loi : l'idée de faire du voyage une activité légalement contrôlée, voire obligatoire, est entièrement inédite. Deuxièmement, un changement tout aussi radical : c'est la première fois que l'idée du voyage d'éducation est évoquée à un niveau collectif. Portiez crée un pont entre la tradition apodémique et les très nombreux projets sur l'éducation qu'a connus le dernier tiers du siècle ${ }^{44}$. Finalement, ce voyage est avant tout identitaire, et ce, au détriment de toute connaissance positive à obtenir - celles-ci ne sont acquises qu'accessoirement. À l'étranger, on rencontre l'imperfection partout; en France, on se penche sur la geste républicaine, à travers la visite de champs de bataille et d'ateliers d'artistes travaillant sur des morceaux célébrant les héros de la république. Tout voyage est une entreprise de connaissance de soi - mais ici, elle atteint un niveau national. Dans cette conception, on se connaît et on se développe avant tout, et en fait exclusivement, en tant que citoyen français, républicain.

«Publier un voyage paraîtra sans doute, au premier coup d'œil, une occupation bien étrangère aux circonstances où se trouve la France ${ }^{45}$.» Le temps n'est pas aux voyages, souligne Brissot de Warville en s'excusant dans la préface de son voyage d'Amérique, ouvrage qu'il publie néanmoins en 1791, du fait de son intérêt pour la situation de la France à ce moment précis.

Mais, en fait, le sujet du voyage ne disparaît pas de la circulation des idées, comme le programme - certes jamais réalisé - de Portiez le montre. Une fois la situation politique normalisée, le voyage refait

44. Robert Palmer estime qu'une centaine de programmes d'éducation ont vu le jour entre le dernier tiers de l'Ancien Régime et les cinq premières années de la Révolution. Robert R. Palmer, The Improvement of Humanity. Education and the French Revolution, Princeton, Princeton University Press, 1985, p. 89.

45. Jacques Pierre Brissot (de Warville), Nouveau voyage dans les États-Unis de l'Amérique Septentrionale, Paris, Buisson, 1791, p. I (première phrase de la préface). 
surface, avec des manifestations aussi disparates qu'un concours poétique de 1807 sur le sujet du «Voyageur » ${ }^{46}$, ou le bien plus important questionnaire de Volney sur l'Amérique (1793), ou celui établi par Joseph-Marie de Gérando pour l'observation des peuples sauvages $(1800)^{47}$.

Justin Stagl place la fin de la tradition apodémique vers 1800 - or, il ne nous semble pas que cette date soit une frontière étanche. Ce qui disparaît, certainement, ce sont les instructions pour le voyage d'instruction à des fins individuelles. Le voyage individualisé subsiste mais, par définition, il voudra et devra être accompli sans « instructions » dans un monde où le rôle de l'individu sera fortement mis en avant.

Mais survivra à sa place le voyage, individuel ou même (comme Portiez le suggère) collectif, au service soit de la science, soit de l'État - le service de la science étant, bien sûr, souvent une forme de service de l'État. Ce processus est bien entamé avant la Révolution, et ne cesse certainement pas à la fin du siècle, ni en France, ni ailleurs. Les instructions pour ce voyage, patriotique ou scientifique, peuvent prendre de nombreuses formes; nous y trouvons parfois même des accents anti-individualistes ${ }^{48}$. Mais dans toutes ces formes, ces instructions représentent une tradition importante, et nettement distincte du nouveau genre qui émerge parallèlement, témoignage et outil du voyage de masse : le guide de voyage.

46. Le vainqueur est Charles Millevoye, poète protégé par l'empereur - voir Le voyageur, pièce qui a remporté le prix décerné par l'Académie française, Paris, A.-A. Renouard, 1807.

47. Sur les Questions de statistique de Volney (1793), voir le chapitre 7 de Justin Stagl: A History of Curiosity, op. cit., "From the Private to the Sponsored Traveller ", p. 269-296; Joseph-Marie de Gérando, Considérations sur les diverses méthodes à suivre dans l'observation des peuples sauvages, s.l.n.d. [Paris, 180o]. Le texte est bien sûr lié à l'activité de la Société des observateurs de l'homme.

48. Ainsi les instructions du baron d'Hauterive pour les jeunes diplomates en mission, dont les rapports devaient servir à la fois l'État et la science de l'État, contiennent des remarques très acerbes sur le fait que la nouvelle jeunesse ne veut pas écouter les instructions données par ses aînés. Alexandre-Maurice Blanc de la Notte, baron d'Hauterive, Quelques conseils à un jeune voyageur, s.l.n.d. [Paris, 1826], p. 20. 